\title{
Bronchial manifestation of acute febrile neutrophilic dermatosis (Sweet's syndrome)
}

\author{
R. Thurnheer*, U. Stammberger**, S. Hailemariam+, E.W. Russi*
}

\begin{abstract}
Bronchial manifestation of acute febrile neutrophilic dermatosis (Sweet's syndrome). $R$. Thurnheer, U. Stammberger, S. Hailemariam, E.W. Russi. CERS Journals Ltd 1998.

ABSTRACT: We describe a $62 \mathrm{yr}$ old female with acute febrile neutrophilic dermatosis (AFND) or Sweet's syndrome, involving the bronchial mucosa.

The patient presented with classical skin manifestations of AFND accompanied by bilateral pneumonia, pleural effusions and lesions of the oral mucosa. The diagnosis was confirmed by skin and mucosal biopsies. Bronchoscopy revealed raised pustules, similar to the lesions of the skin, suggesting bronchial involvement of AFND. The patient responded to systemic corticosteroids only for a short period and died as a result of multiorgan failure. Postmortem findings confirmed dense, focal neutrophilic infiltrates of the bronchi and bronchioles. No underlying disease was found.

Eur Respir J 1998; 11: 978-980.
\end{abstract}

Division of Pulmonary Medicine, *Dept of Internal Medicine, **Dept. of Surgery, and +Institute of Clinical Pathology, University of Zürich, Switzerland.

Correspondence: E.W. Russi, Division of Pulmonary Medicine, Dept of Internal Medicine, Rämistr. 100, 8091 Zürich, Switzerland, Fax: 4112554451

Keywords: Acute febrile neutrophilic dermatosis, bronchial involvement, Sweet's syndrome,

Received: 30 January 1997

Accepted after revision 8 June 1997
Acute febrile neutrophilic dermatosis (AFND) or Sweet's syndrome, was first described in 1964 by SwEET [1]. The disorder was initially characterized as an acute eruption of painful erythematous plaques and nodules, associated with fever, leukocytosis and dense papillary dermal neutrophilic infiltrates evident on biopsy specimen. Subsequently, various lesions, including erythematous plaques and painful papules with pseudoblistering and occasional pustules and bullae have been reported [2].

The syndrome typically occurs in middle-aged females after a nonspecific infection of the respiratory or gastrointestinal tract, or in association with haematopoietic disorders, inflammatory or malignant diseases [3-6]. STORER et al. [7] have shown an association between AFND and Sjögren's syndrome, ulcerative colitis, metastatic adenocarcinoma and testicular carcinoma.

AFND has been observed involving the eyes, joints, oral mucosa and organs such as the lung, the liver, the kidneys and the central nervous system [3]. The aetiology of AFND remains unknown. Some investigators suggested that the condition represents a variable manifestation of hypersensitivity to bacterial or chemical allergens $[8,9]$. Pulmonary manifestations of AFND, consisting of bilateral infiltrates, bronchiolitis obliterans-organizing pneumonia (BOOP) and pleural effusions have been described elsewhere. We report a patient with AFND involving the lungs as well as the bronchial tree.

\section{Case report}

A $62 \mathrm{yr}$ old female was admitted with a 14 day history of painful skin lesions involving the legs, face, arms and trunk. Initially, the lesions consisted of tender and painful erythematous plaques and nodules, with some blistering or formation of pustules. Later, grouped pustular lesions were also present on the soft palate. Six days after the occurrence of the skin lesions, fever and chills developed. Tetracycline and oral steroids (50, 25 and $12.5 \mathrm{mg} \cdot$ day $^{-1}$ of prednisone were administered on day 1 , days $2-3$, and days $4-5$, respectively) were given as a result of history of a tick bite incurred 8 days previously. Fever resolved within $24 \mathrm{~h}$ but reappeared after discontinuation of the oral steroids 4 days later. Right-sided chest pain developed and the chest radiograph showed bilateral dense infiltrates of the lower lobes (fig. 1). On admission, total

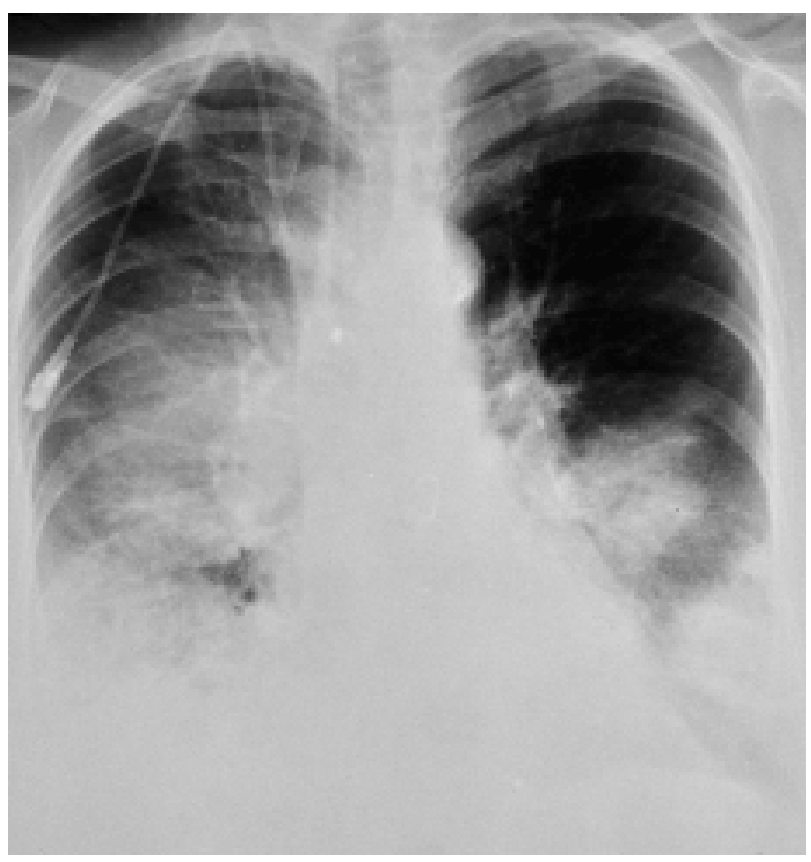

Fig. 1. - Chest radiograph showing areas of ill-defined consolidation affecting particularly the right-lower and mid-zones and the left-lower zones. 
leukocyte count was 9,120 cells $\mu \mathrm{L}^{-1}$ (84\% neutrophils). Serum protein electrophoresis revealed slightly decreased albumin and normal $\gamma$-globulin levels. The liver enzymes were normal. Fibreoptic bronchoscopy (FB) with bronchoalveolar lavage (BAL) were performed, total cell count was 3,100 cells $\cdot \mu \mathrm{L}^{-1}$ (78\% neutrophils, $14 \%$ macrophages and $8 \%$ lymphocytes).

No bacteria were found by microscopy in the BAL and cultures for mycobacteria, fungi and viruses (Cytomegalo-, Herpes-, Epstein-Barr-, Adeno-, Influenza A/B-, Parainfluenza $1 / 2 / 3$-virus) remained negative. The trachea was normal but multiple pustules were found on the mucosa of the right and left mainstem bronchi (fig. 2). They were of the same aspect as those in the oral cavity, histologically shown to consist of neutrophilic infiltrates in the upper mucosal layers (fig. 3). There was no evidence of vasculitis, and immunofluorescence studies gave negative results. The fluid of the mucosal pustule contained mature polymorphic neutrophils but no micro-organisms, and cultures

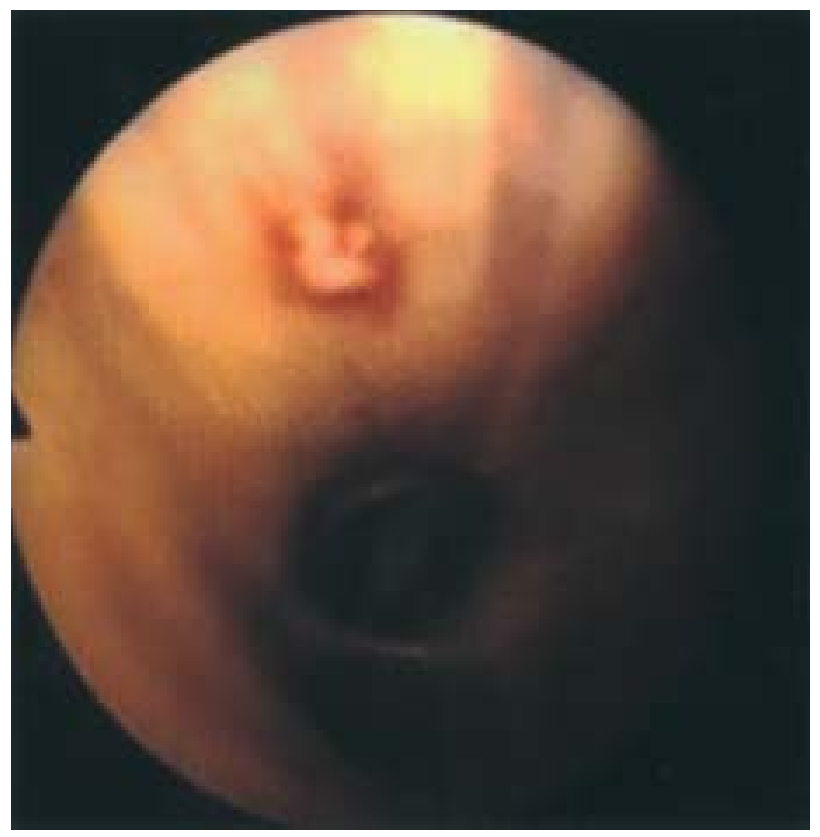

Fig. 2. - Fibreoptic view of the left main bronchus demonstrating one raised pustule with a reddish border.

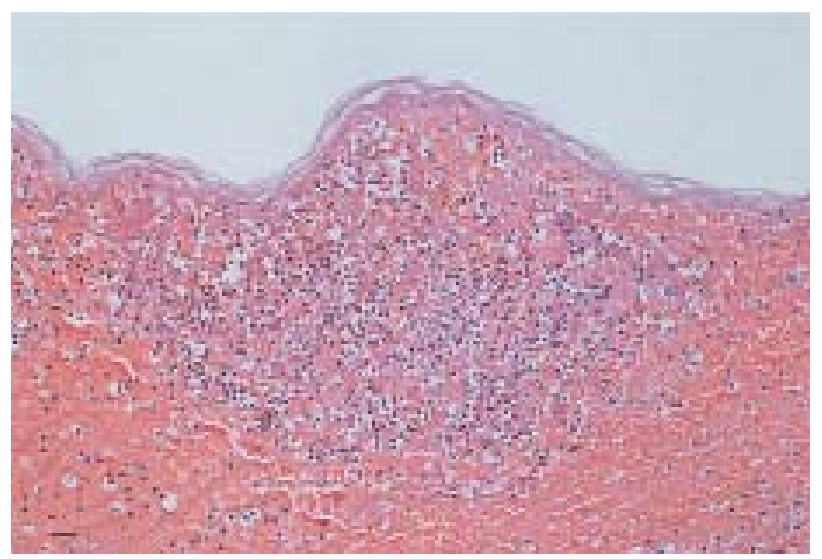

Fig. 3. - Buccal mucosa with the same aspect as in the oral cavity, histologically shown to consist of neutrophil infiltrates in the upper mucosal layer. (Haematoxylin stain; internal scale bar $=50 \mu \mathrm{m}$ ).
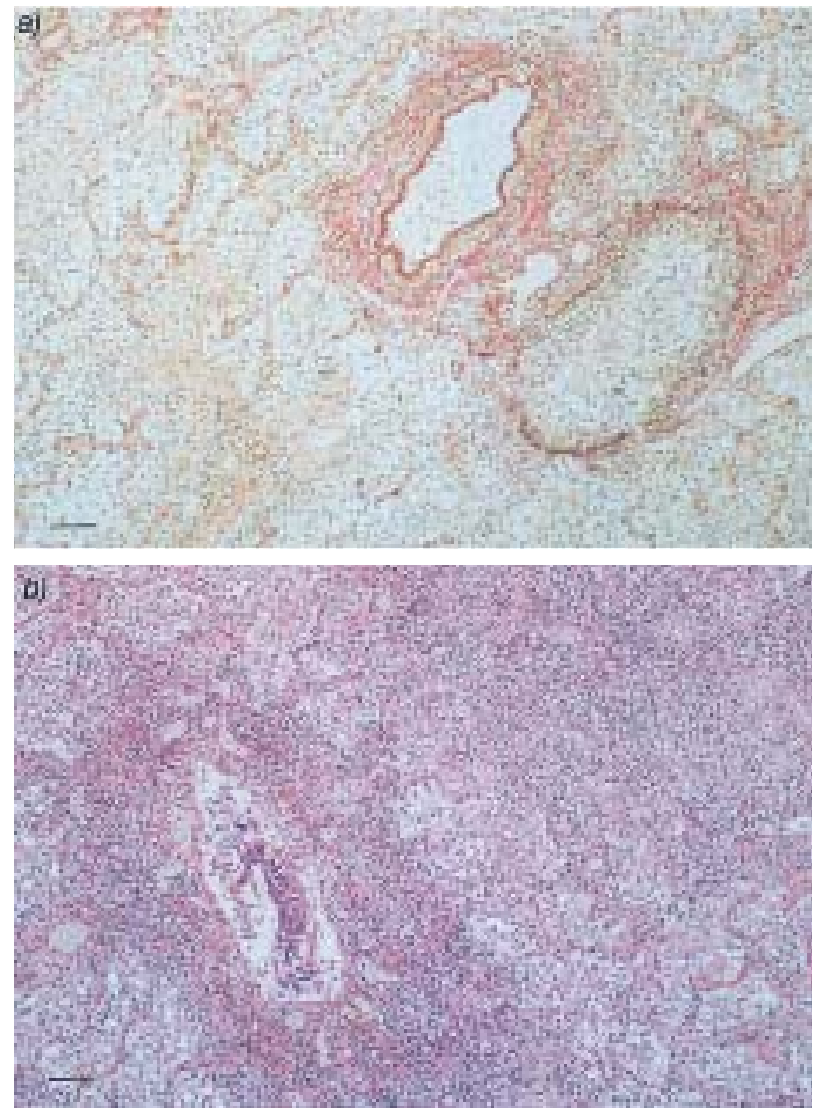

Fig. 4. - Microscopic appearance of the lung obtained by autopsy, showing granulocytic infiltration of the bronchiolar wall, alveolar wall thickening with interstitial granulocytic infiltration and an intraalveolar neutrophil infiltrate. (a) Haematoxylin stain and (b) van Grieson stain; internal scale bar $=50 \mu \mathrm{m}$.

for Herpes virus were negative. Repeated blood cultures were also negative.

As a result of severe thrombocytopenia, no biopsies were taken from the bronchial mucosa. The patient was treated with parenteral antibiotics with no clinical improvement. Bone marrow biopsy and aspiration revealed nonspecific alterations of myelopoietic cells but no haematological malignancy. No malignancy was found by computed tomography of the thorax, abdomen and pelvis. After 5 days of unsuccessful antibiotic treatment, the patient was administered parenteral prednisone $\left(100 \mathrm{mg} \cdot\right.$ day $\left.^{-1}\right)$ Her condition improved, but 4 days later, she developed acute respiratory distress syndrome (ARDS) and died as a result of multiorgan failure. A postmortem work-up showed interstitial infiltrates of widened alveolar septa as well as alveolar neutrophilic exudate. Dense focal granulocytic infiltrates of the bronchial wall were also present (fig. 4).

\section{Discussion}

Neutrophilic dermatoses are dermatological conditions characterized predominantly by polymorphonuclear infiltrates. They include Sweet's Syndrome, pyoderma gangrenosum and subcorneal pustular dermatosis, among others [10]. 
By definition, febrile neutrophilic dermatosis comprises the following cardinal features: fever; polymorphonuclear leukocytosis, raised painful plaques on the extremities, face, and neck; and a dense dermal cellular infiltrate with mature neutrophils on histology [1]. In 1986, Su and LIU [11] proposed two major and four minor criteria to serve as a guide for the diagnosis of AFND (table 1). It has since been established that both major and two of the minor criteria are prerequisite for diagnosis. According to these guidelines, our patient met the two major and three of the minor criteria.

Pulmonary involvement of AFND was first described in 1985 by GiBSon et al. [12]. Only a few case reports of biopsy-proven pulmonary involvement have been recorded since [13-15]. In the largest case series of 48 retrospectively-reviewed cases presented at Mayo Clinic between 1980 and 1995 [16], five patients had concomitant pulmonary infiltrates, and all were responsive to corticosteroids but not to antibiotics. Similar pulmonary infiltrates have been described in the course of Pyoderma gangrenosum, another neutrophilic dermatosis [17]. In our case, there were skin and oral lesions, which were histologically confirmed as neutrophilic dermatosis, and were accompanied by similar macroscopic changes in the bronchi. Thorough investigation for a potential aetiology remained negative. Repeated viral cultures including Herpes virus were negative. No other micro-organism and no neoplastic disorder was detected. Extensive pulmonary infiltrates have been proposed to be due to neutrophilic alveolitis based on BAL findings, but no lung biopsy was performed. Pulmonary infiltrates progressed despite antibiotic treatment. Fever and acute phase protein abated transiently, but the patient subsequently developed ARDS and died as a result of multiorgan-failure.

FETT et al. [16] described five patients suffering from AFND with pulmonary involvement. Two patients improved, two showed recurrent cutaneous lesions, and one died after an initial improvement on steroid therapy.

Observations with regards to aspect, skin and oral lesions were thought to resemble those of bullous pemphigoid or Stevens-Johnson syndrome. However, the negative immunological stains and the intact epidermis in the skin biopsy sections are not consistent with either diagnosis $[17,18]$.

Our observations strongly suggest that acute febrile neutrophilic dermatosis may also involve the mucous mem-

Table 1. - Criteria for the diagnosis of acute febrile neutrophilic dermatosis

\footnotetext{
Major criteria

Abrupt onset of tender or painful erythematous or violaceous plaques or nodules and

Predominantly neutrophilic infiltration in the dermis without leukocytoclastic

Minor criteria

Preceded by fever or infections

Accompanied by fever, arthralgia, conjunctivitis, or underlying malignancy

Leukocytosis

Good response to systemic steroids and not to antibiotics

According to $\mathrm{Su}$ and Lu [11].
}

branes of the bronchial tree. Histologically, the bronchial inflammation resembles that seen in skin and mucosal lesions, consisting predominantly of mature neutrophils infiltrating the interstitium in the absence of pathogenic organisms.

Acknowledgment: The authors would like to thank B. Krayenbühl and B. Müller, Dept of Dermatology, University Hospital of Zurich for providing pictures and comments regarding the histopathology of the skin.

\section{References}

1. Sweet RD. An acute febrile neutrophilic dermatosis. $\mathrm{Br} J$ Dermatol 1974; 76: 349-356.

2. Von den Driesch P. Sweet's syndrome (acute febrile neutrophilic dermatosis). J Am Acad Dermatol 1994; 31: 535-556.

3. Cohen PR, Talpaz N, Kurzrock R. Malignancy-associated Sweet's syndrome: review of the world literature. J Clin Oncol 1988; 6: 1887-1897.

4. Cooper PH, Innes DJ Jr, Greer KE. Acute febrile neutrophilic dermatosis (Sweet's syndrome) and myeloproliferative disorders. Cancer 1983; 51: 1518-1526

5. Klock JC, Oken RL. Febrile neutrophilic dermatosis in acute myelogenous leukemia. Cancer 1976; 37: 922-927.

6. Spector JI, Zimbler H, Levine R, Ross JS, Valigorsky JM, Cole LM. Sweet syndrome-Association with acute leukemia. JAMA 1980; 4: 283-288.

7. Storer JS, Nesbitt LT, Galen WK, De Leo VA. Sweet's syndrome - a review. Int J Dermatol 1983; 22: 8-12.

8. Wenning J. Akute febrile neutrophile Dermatosen- Sweetsyndrom. Hautarzt 1978; 29: 467.

9. Fuld H. New dermatosis (letter to the editor). Br Med J 1965; 1: 382.

10. Vignon-Pennamen, MD, Zelinsky-Gurung A, Janssen F, Fija J, Wallach D. Pyoderma gangrenosum with pulmonary involvement. Arch Dermatol 1989; 125: 1239-1242.

11. Su D, Liu H, Diagnosic criteria for Sweet's syndrome. Cutis 1986; 37: 167-170.

12. Gibson LE, Dicken CH, Flach DB. Neutrophilic dermatosis and myeloproliferative disease: report of two cases. Mayo Clin Proc 1985; 60: 735-740.

13. Lazarus AA, Mc Millan M, Miramadi A. Pulmonary involvement in Sweet's syndrome (acute febrile neutrophilic dermatosis). Chest 1986; 90: 922-924.

14. Takimoto CH, Warnock M, Golden JA. Sweet's syndrome with lung involvement. Am Rev Respir Dis 1991; 143: 177-179.

15. Bourke SJ, Quinn AG, Farr PM, Ashcroft T, Gibson GJ. Neutrophilic alveolitis in Sweet's syndrome. Thorax 1992; 47: 572-573.

16. Fett DL, Gibson LE, Su WPD. Sweet's syndrome: systemic signs and symptoms and associated disorders. Mayo Clin Proc 1995; 70: 234-240.

17. Perl S, Rappersberger K, Fodinger D, Anegg B, Honigsmann H, Orter B. Bullous pemphigoid induced by PUVA therapy. Dermatology 1996; 193: 245-247.

18. Rzany B, Hering O, Mockenhaupt M, et al. Histopathological and epidemiological characteristics of patients with erythema exudativum multiforme major, StevensJohnson syndrome and toxic epidermal necrolysis. $\mathrm{Br} J$ Dermatol 1996; 135: 6-11. 